\title{
Understanding the mechanisms of cooperative physico-chemical treatment and mechanical disintegration of biomass as a route for enhancing enzyme saccharification
}

\author{
Roger Ibbett $^{1}$ (D) $\cdot$ Sanyasi Gaddipati ${ }^{1} \cdot$ Greg Tucker $^{1}$
}

Received: 5 April 2017 /Revised: 10 August 2017 / Accepted: 6 October 2017 /Published online: 31 October 2017

(C) The Author(s) 2017. This article is an open access publication

\begin{abstract}
A novel chemico-kinetic disintegration model has been applied to study the cooperative relationship between physico-chemical treatment and supplementary wet-state milling of biomass, as an efficient process route to achieve high enzyme accessibility. Wheat straw, Miscanthus and shortrotation willow were studied as three contrasting biomass species, which were subjected to controlled hydrothermal pretreatment using a microwave reactor, followed by controlled wetstate ball-milling. Comparative particle disintegration behaviour and related enzyme digestibilities have been interpreted on the basis of model parameters and with evaluation of textural and chemical differences in tissue structures, aided by the application of specific material characterisation techniques. Supplementary milling led to a $1.3 \times, 1.6 \times$ and $3 \times$ enhancement in glucose saccharification yield after $24 \mathrm{~h}$ for straw, Miscanthus and willow, respectively, following a standardised 10-min hydrothermal treatment, with corresponding milling energy savings of 98,97 and $91 \%$ predicted from the model, compared to the unmilled case. The results confirm the viability of pretreatment combined with supplementary wet-milling as an efficient process route. The results will be valuable in understanding the key parameters for process design and optimisation and also the key phenotypical parameters for feedstock breeding and selection for highest saccharification yield.
\end{abstract}

Keywords Biomass · Disintegration $\cdot$ Pretreatment . Modelling $\cdot$ Saccharification

Roger Ibbett

roger.ibbett@nottingham.ac.uk

1 School of Biosciences, University of Nottingham, Loughborough LE12 5RD, UK

\section{Introduction}

The efficient physico-chemical deconstruction of biomass as a pretreatment for enzyme saccharification requires optimisation of a number of factors, including the extent to which the components of the cell wall are debonded by pretreatment reactions [1-4], the accessibility of the cellulose fibrils within the debonded tissue structures towards enzyme hydrolysis [5, 6] and the extent to which the accessibility of these residual tissue structures evolves due to breakup during physical processing [7-9]. Overall resistance to deconstruction may therefore depend on a number of phenotypical attributes, which might be targeted for process optimisation or by feedstock selection [10-13].

One significant attribute of a biomass feedstock is the mechanical durability of the tissue structure, which will determine the ease of disintegration by mechanical processing. Such techniques are designed to bring about an increase in accessible surface area, which in turn leads to an enhancement in enzyme digestion rate and yield. Previous studies have shown that dry ball-milling of untreated biomass can be effective in this regard $[14,15]$ although this may be a lengthy and energy-intensive operation $[16,17]$. Wet-state mechanical disintegration is more advantageous in that it avoids the energy expense of drying and can be integrated into a sequence of other wet processing steps, either as a pretreatment $[18,19]$ or combined with simultaneous enzymatic treatment $[20,21]$. Furthermore, studies have also shown that the energy consumption of wet-milling and related disintegration methods can be reduced significantly by the application of a prior chemical treatment, either in sequence or as part of a combined process [22-24]. Such cooperative methodologies may be key in achieving high enzyme digestibility at economic process cost $[15,25]$. Also importantly, the supplementary application of wet-milling following pretreatment has the potential to achieve good saccharification yields from more 
recalcitrant biomass species, such as woody materials, potentially with reduced severity of the pretreatment step [23]. Lower severities would also reduce unwanted biomass degradation, limiting the production of compounds that inhibit fermentation and minimising the loss of useful sugars, improving overall yield [26].

Following these previous key findings, this current work sets out to understand more fully the influence of prior physico-chemical treatment on the rate of mechanical disintegration of a biomass feedstock, by a new application of a chemico-kinetic model for material failure. This model was originally developed to predict the failure of fibres and polymers under a fatigue regime [27, 28], and has also been adapted to explore the influence of cross-linking on the wet abrasion of cellulose fibres [29]. For this current study, a further modification of the model has been developed, to incorporate terms describing the extent of disruption of molecular bonding caused by chemical pretreatment, which will then modify the rate of mechanical disintegration under milling type conditions. With the aid of the model, this new study has for the first time directly contrasted the behaviour of three commercially important biomass species, namely wheat straw, Miscanthus and short-rotation willow, subjected to highly controlled pretreatment and milling regimes. The impact of tissue architecture and cellular material differences between biomass species has been interpreted in terms of the disintegration model parameters, supported by data from specific material characterisation techniques. Treated biomass materials have been assessed using an established enzyme hydrolysis assay, in order to correlate digestion with disintegration performance.

\section{Theory and modelling}

Any solid material, either synthetic or of natural origin, gains resilience against repetitive mechanical action from its network of molecular bonds, which resist external stresses and maintain material integrity. In the Zhurkov kinetic model, it is considered that the molecular bonds in a material are constantly undergoing fluctuations in length due to natural thermal motion, and that as a result of these statistical fluctuations, over the course of time, some bonds may exceed their stress limit and will undergo rupture [27]. Moreover, the application of external stresses, say due to milling or abrasion, will add to the stress experienced at the molecular level, so that the chance of thermal fluctuations exceeding the stress limit will increase and ruptures will occur over a shorter time period. The progressive, time-dependent rupture of individual connecting bonds in a material leads eventually to macroscopic failure and the creation of new fracture surfaces, which in the case of milling results in a reduction in particle size and an increase in surface area.
The time to a defined point of macroscopic failure will depend inversely both on the temperature and also the average magnitude of the applied stress under cyclic or rotatory milling conditions, where both parameters influence the statistical chance of bond fluctuations exceeding the stress limit, according to an Arrhenius principle. The time to failure also depends on the morphology and molecular organisation of the material, which in the model can be represented by a material integrity parameter. This will vary between biomass species and is the main descriptor of material properties, which will report on both cellular architecture as well as the molecular organisation within the cell wall. In this new adaptation of the model, it is also appreciated that the value of this integrity parameter will be reduced if a proportion of linkages in the biomass material have been broken due to prior physico-chemical treatment, as illustrated in Fig. 1a. From previous studies, the extent of the deconstruction reactions due to hydrothermal pretreatment can be defined by applying standard chemical kinetics [30].

The complete model for time-dependent failure under milling or abrasion conditions is set out in Eq. (1), where $\tau$ is the critical time required for size reduction by wet ball-milling in order to create the necessary material surface area for effective enzyme accessibility.

$\tau=\tau_{0} \exp \left[\frac{U_{0}-\frac{\sigma}{m(1-f)}}{R T}\right]$

$f=1-\exp (-k t)$

In Eq. (1), $\tau_{0}$ is a proportionality constant, which is defined as the time to failure at infinite temperature, related to the timescale for molecular vibrations. $U_{0}$ is the unperturbed activation energy for the molecular rupture process, $\sigma$ is the average external stress resulting from cyclic mechanical action, $R$ is the gas constant and $T$ is the absolute temperature. The material integrity parameter, $m$, formally has units representing the initial number of moles per unit volume of stress-active bonds. This is modified by $f$, the extent of the physico-chemical deconstruction reactions, as described by first-order chemical kinetics, according to Eq. (2), where $t$ is the pretreatment time and $k$ is a rate constant. For this work, in Eq. (1), a value of $100 \mathrm{~kJ} \mathrm{~mol}^{-1}$ was used for $U_{0}$, and $10^{-12} \mathrm{~s}$ for $\tau_{0}$. A value of $0.02 \mathrm{GPa}$ was used for $\sigma$, for a mechanical regime consisting of shear and compressional type cyclic fatigue, estimated according to earlier studies [29]. Fitting of the model to experimental data was achieved by minimisation of the sum of squared differences, using a combination of manual iteration and the Solver ${ }^{\mathrm{TM}}$ tool in Microsoft Excel. 
a

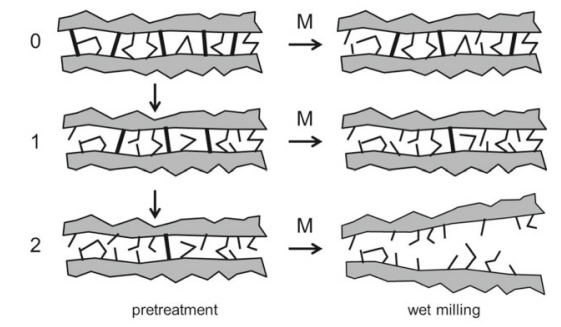

b

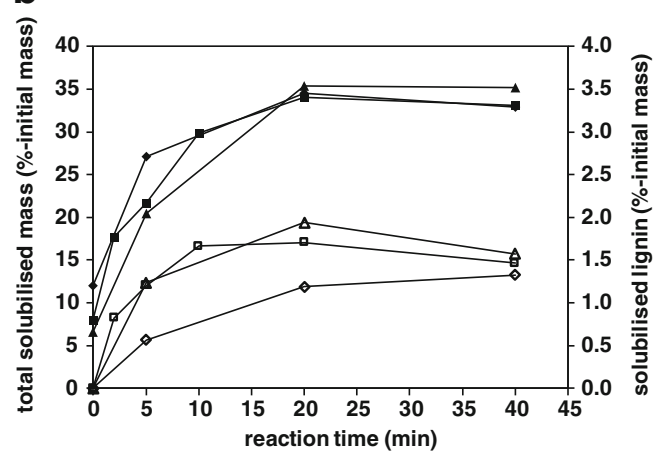

Fig. 1 a Schematic of chemico-kinetic model for biomass disintegration. In the original state, a proportion of active bonds share the stress. Both active bond (thick lines) and inactive bonds (thin lines) are reduced in number by prior chemical treatment, from levels 0 to 1 to 2 , where the active bonds are further disrupted by a milling time of $\mathrm{M}$. $\mathbf{b}$ Solubilisation of material following hydrothermal treatment at $200{ }^{\circ} \mathrm{C}$ for increasing reaction times, expressed as percentage of original total biomass: total solubilised mass from wheat straw (filled square), Miscanthus (filled triangle) and willow (filled diamond); solubilised lignin from wheat straw (empty square), Miscanthus (empty triangle) and willow (empty diamond)

\section{Experimental materials and methods}

\subsection{Plant materials}

Wheat straw (var. Cordiale) was obtained from the University of Nottingham farm. Miscanthus (var. Giganteus) was provided by the University of Aberystwyth. Short-rotation willow (var. Tora) was provided by Rothamsted Research Station. All feedstocks were air dried to ambient laboratory moisture content and were stored for approximately 3 months before use. Prior to treatments, all feedstocks were knife milled to pass through a coarse 2-mm screen (model P90 mill, Fritsch GmbH).

\subsection{Hydrothermal pretreatment}

Samples of $2 \mathrm{~g}$ of untreated knife-milled biomass were each loaded into 30-ml glass microwave reactor vials with $20 \mathrm{ml}$ of water. After 30 min equilibration, each vial was sealed and placed in the chamber of a microwave reactor (Monowave 300, Anton Paar $\mathrm{GmbH}$ ). The maximum rate of heating was applied, enabling a set point of $200{ }^{\circ} \mathrm{C}$ to be reached in $90 \mathrm{~s}$, under control of a ruby fluorescence thermometer. The set temperature was held for different isothermal periods, from 1 to 20 or $40 \mathrm{~min}$, followed by rapid cooling to ambient temperature by forced air circulation. Untreated control samples of each biomass species were subjected to a lower temperature sterilisation at $121{ }^{\circ} \mathrm{C}$ for $5 \mathrm{~min}$, in order to neutralise any potential microbial action that might develop during subsequent enzyme hydrolysis, but without altering the biomass structure. For gravimetric determinations, the treated samples were washed four times through a paper filter with demineralised water, and the solid residues were air dried, then oven dried at $100{ }^{\circ} \mathrm{C}$, to establish the proportional weight losses due to solubilisation of cell wall species [31]. For samples prepared for enzyme digestion experiments, the same washing protocol was applied but with overnight drying at ambient temperature, to constant weight, in order to limit any possible structural collapse (hornification) which might influence subsequent enzyme accessibility. For modelling of wet-milling behaviour, after treatment, the total contents of the reactor vials were transferred without washing to ball-mill vessels. For microscopy investigations, cut stem sections of straw, Miscanthus and willow were hydrothermally treated in the microwave reactor at $200{ }^{\circ} \mathrm{C}$ in excess water, and the treated samples were recovered without damage.

\subsection{Chemical analysis}

The as-received biomass materials and selected washed and dried pretreated residues were subjected to total acid hydrolysis by incubating for $1 \mathrm{~h}$ in $12 \mathrm{M}$ sulphuric acid at $37^{\circ} \mathrm{C}$, followed by $2 \mathrm{~h}$ in $1 \mathrm{M}$ sulphuric acid at $98^{\circ} \mathrm{C}$ [32]. Sugar monomer concentrations in the acid hydrolysates were determined by high-performance ion-exchange chromatography with pulsed amperometric detection (Dionex, UK) [30]. Analysis of lignin in the as-received biomass and the washed and dried pretreated residues was carried out by extraction using acetyl bromide in acetic acid, followed by measurement of the absorbance at $280 \mathrm{~nm}$ [33]. Lignin quantification was performed by calibration using a low sulphate lignin reference sample (Sigma-Aldrich Ltd.).

\subsection{Wet-milling procedures}

As outlined above, in one procedure, the total liquors and solids following pretreatment were directly transferred to 80 $\mathrm{ml}$ stainless steel ball-mill vessels, with total liquor volumes made up to $50 \mathrm{ml}$ by washing with $28 \mathrm{ml}$ of demineralised water (giving a final $4 \%$ solids content); $15 \times 1$-cm-diameter steel balls were added to each vessel, and the lids were sealed with PTFE gaskets (P9 planetary ball mill, Fritsch $\mathrm{GmbH}$ ). Milling was carried out for differing total times at $250 \mathrm{rpm}$, comprising of cycles of $2 \mathrm{~min}+2 \mathrm{~min}$ pauses. This procedure was applied to a set of willow biomass samples which had been subjected to increasing pretreatment times at $200{ }^{\circ} \mathrm{C}$, which were then each milled for a total of $10 \mathrm{~min}$. The milled 
willow dispersions were assessed by microscopy and by sedimentation protocols, as described below. The same milling procedure was applied to sets of samples of each of the three biomass species, which had again been pretreated for increasing times at $200{ }^{\circ} \mathrm{C}$. Each of these samples was milled for a series of accumulated time steps, with assessment at each increment to establish the critical milling time $(\tau)$ required to achieve a given extent of particle breakup. This was assessed rheologically by determining the total milling time required to reduce the particles to a sufficiently small size for the dispersions to flow easily under suction through a 1-mm-diameter capillary pipette. The operation was repeated twice for each biomass, giving measurements in duplicate.

In a separate procedure, $200 \mathrm{mg}$ portions of washed and ambient dried samples of the three biomass species were pretreated for different times at $200{ }^{\circ} \mathrm{C}$ and were subjected to wet ball-milling using the above machine and vessel parameters for selected times. For this procedure, the milling liquor consisted of $36 \mathrm{ml}$ of a $50-\mathrm{mM} \mathrm{pH} 5$ citrate buffer solution, so that the milled dispersions could be transferred directly to $50-\mathrm{ml}$ centrifuge tubes for a standardised enzyme hydrolysis assay. This wet transfer was designed to avoid material transfer losses, and also to avoid an additional drying step which might cause structural changes and which might influence enzyme accessibility. All samples subjected to the enzyme assay had therefore experienced the same single drying step, so all comparisons were considered internally consistent. A 4-ml volume of enzyme concentrate in $\mathrm{pH} 5$ buffer was added to the dispersions in the centrifuge tubes, giving a final $0.5 \%$ solids content, for the digestion protocol described below. A zero milling time sample was also included in each series. For calculations, the initial sample weights were corrected for equilibrium moisture. Sedimentation experiments were also carried out on the intermediate $10 \mathrm{~min}$ pretreated dispersions in the $50-\mathrm{ml}$ tubes, prior to the enzyme assay, as described below. An equivalently treated set of dried samples of each biomass were characterised by wide-angle X-ray diffraction and also examined by optical microscopy.

\subsection{Physical characterisation}

Transmitted light optical microscopy images of biomass particles dispersed in water. Scanning electron microscopy images of gold-coated samples of untreated and treated biomass were obtained using a Hitachi S300 instrument, at $13 \mathrm{kV}$. In addition, optical micrographs of dispersions diluted to $0.08 \%$ were processed using ImageJ software (ImageJ 1.45, http://imagej.nih.gov.i), to estimate the distribution of particle diameters and volumes, assuming spherical geometry.

Material densities of the untreated biomass materials were determined by helium pycnometry (Quantachrome Inc), using 2-mm-cut-size samples. Hydrated bulk densities of the untreated 2-mm-size biomass samples were determined by measurement of the volume displacement of $4 \mathrm{~g}$ of material immersed in $30 \mathrm{ml}$ of demineralised water, after $24 \mathrm{~h}$ hydration. The settling characteristics of the dispersions of biomass produced by wet-milling were determined in $50-\mathrm{ml}$ centrifuge tubes, both for 4 and $0.5 \%$ solids samples. The dispersions were shaken and allowed to settle over $24 \mathrm{~h}$ at ambient temperature, ensuring no floating particles. The final volume of the lower particulate phase was measured as a fraction of the total volume of the sample.

Wide-angle X-ray diffractograms of untreated, pretreated and wet-milled materials were measured using a Siemens S5000 system, as described previously [34]. An amorphous subtraction procedure was carried out using extensively dry ball-milled samples from each biomass species. Considering cellulose as the only crystallisable polymer in the cell wall matrix, an estimation of the cellulose fibril crystallinity could be established by division of the total crystallinity by the fraction of glucan in the sample, obtained by sugar analysis. This approach assumed that all glucan was derived from the cellulose component, neglecting the small amount of glucan that might be present in the hemicellulose components.

\subsection{Enzyme digestion and saccharification yields}

Portions of $200 \mathrm{mg}$ of biomass substrates were prepared as described above by milling as dispersions in $36 \mathrm{ml}$ of pH 5 citrate buffer. Incubations were carried out in 50-ml centrifuge tubes in the presence of $40 \mathrm{FPU} / \mathrm{g}$-biomass of a Trichoderma reesi cellulase mixture (C8546 lyophilised powder, Sigma Aldrich Co Ltd), made up to $40 \mathrm{ml}$ total liquor. The centrifuge tubes were shaken horizontally at $150 \mathrm{rpm}$ in an incubator at $50{ }^{\circ} \mathrm{C}$, with $100-\mu \mathrm{l}$ samples taken at selected time intervals for sugar monomer analysis [34]. Glucose saccharification yields were expressed as a percentage of total glucose (as glucan) in the undigested material.

In addition, the kinetics of enzyme hydrolysis of the biomass samples subjected to the intermediate 10-min hydrothermal treatment were analysed from a full time series using a pseudo-first-order diffusion-limiting model, which is applicable for reactions in heterogeneous substrates tending to a high degree of completion [35]. The experimental glucose yield data was fitted to Eq. (3), where $C_{t}$ is the yield at time t, $C_{\infty}$ is the yield at infinite time, $r$ is a rate constant including both reaction and physical-diffusional contributions, and $n$ is a parameter describing the structural diffusional constraint.

$C_{t}=C_{\infty}[1-\exp (-r t)]^{n}$ 


\section{Results and discussion}

\subsection{Experimental pretreatment and wet-milling of comparative biomass species}

The proportions of cellulose, hemicellulose and lignin and other compositional data for the three biomass feedstocks are summarised in Table 1. The solubilisation profiles for each biomass in Fig. 1b describe the progress of the collective deconstruction reactions of the polysaccharide and lignin cell wall components under hydrothermal conditions. The data was used to derive trial kinetic parameters for application of the disintegration model described in Section 2, where fitting to Eq. (2) gave experimental pretreatment rate constants $(k)$ for straw $=0.19(0.03) \mathrm{min}^{-1}$, Miscanthus $=0.17(0.03) \mathrm{min}^{-1}$ and willow $=0.23(0.04) \mathrm{min}^{-1}$ (errors in parentheses). A greater amount of immediately extractable material was liberated from willow, compared to straw and Miscanthus, due to the solubilisation of sap components trapped in the willow tissue on drying. The data therefore indicated that proportionally less cell wall material was solubilised from willow than Miscanthus or straw under these hydrothermal conditions. This was also consistent with the slower rate of loss indicated from the lignin assay, which is an expected consequence of the higher lignin cross-linking in the wood compared to the two grass species [36].

The electron microscopy images in Fig. 2 show that without mechanical action, the tissue organisation in all three species was retained and even consolidated after pretreatment, despite the chemical transformations of lignin and solubilisation of hemicellulose components in the cell wall. However, the underlying loss of integrity of these tissue structures is demonstrated for willow, in Fig. 3, where an increase in the duration of hydrothermal treatment led to a corresponding increase in extent of tissue breakup after a standard milling time. This was shown by particle size image analysis and also from the settling characteristics of the milled biomass dispersions, where the observed increase in volume of the settled phase resulted from the reduction in size of the milled particles and their more effective suspension within the liquor phase.

\subsection{Modelling of disintegration and relation to material properties}

The disintegration model provides a basis for understanding the cooperative effect of pretreatment and wet-milling on biomass breakup behaviour. The in situ measurement of the capillary flow characteristics of dispersions was chosen as the most practical technique for immediate estimation of particle disintegration, where, as described, the critical milling time $(\tau)$ of the model was defined as the milling time required to achieve smooth flow through a 1-mm-diameter orifice.
Applying this technique, the experimental data in Fig. 4a confirmed that for all three biomass species, there was a strong inverse relationship between the duration of hydrothermal treatment and the resulting critical milling time, as biomass cell wall integrity was reduced by prior deconstruction reactions. However, important differences in behaviour were seen between the three biomass species, which was interpreted through evaluation of model parameters and biomass physical properties, as discussed below.

The trends of the experimental profiles in Fig. 4a serve to validate the utility of the disintegration model. Selected hypothetical model outputs in Fig. 4b successfully predict that the critical milling time for a material reduces with the extent of pretreatment, and that for a given extent of pretreatment, a material with a higher material integrity parameter will require a longer critical milling time to achieve the desired size reduction. However, a deficiency of the model as set out in Eqs. (1) and (2) is that the critical milling time tends to zero at long pretreatment times, implying that the treated biomass breaks up instantaneously. However, the experimental data from Fig. 4a showed that a measureable milling time was still required to achieve the critical size reduction, even at the longest pretreatment times. From the kinetic profiles in Fig. 1b, the primary deconstruction reactions were complete after $20 \mathrm{~min}$ hydrothermal pretreatment at $200{ }^{\circ} \mathrm{C}$, as hemicellulose and lignin components reached a limit in solubilisation. However, cellulose is more resistant to hydrothermal reactions at these treatment temperatures, and also much of the lignin material remains insoluble, potentially recondensing into a new network. Therefore, some structural integrity would be expected to remain, which may be accounted for by a modification of the reaction terms in the model Eq. (2). The measured pretreatment rate constant $k$ is therefore replaced by $k^{\prime}$, which is a revised mean rate constant accounting for material reacting by other pathways than detected through solubilisation. The extent of reaction $f$ is also replaced by $f^{\prime}$, giving a revised first-order kinetic Eq. (4), where $p$ is a scaling factor accounting for the possibility of a proportion of the biomass being chemically resistant or remaining stable through the hydrothermal treatment. Both these revised parameters were optimised via simultaneous fitting to the disintegration model. With these adjustments, it was possible to achieve good fits to the experimental data at all pretreatment times, shown as continuous lines in Fig. 4a, with the final fitted model parameters for each biomass type summarised in Table 2.

$f^{\prime}=\left[1-\exp \left(-k^{\prime} t\right)\right] p$

In support of this modification of the model, the experimental results revealed that the critical milling time for willow 


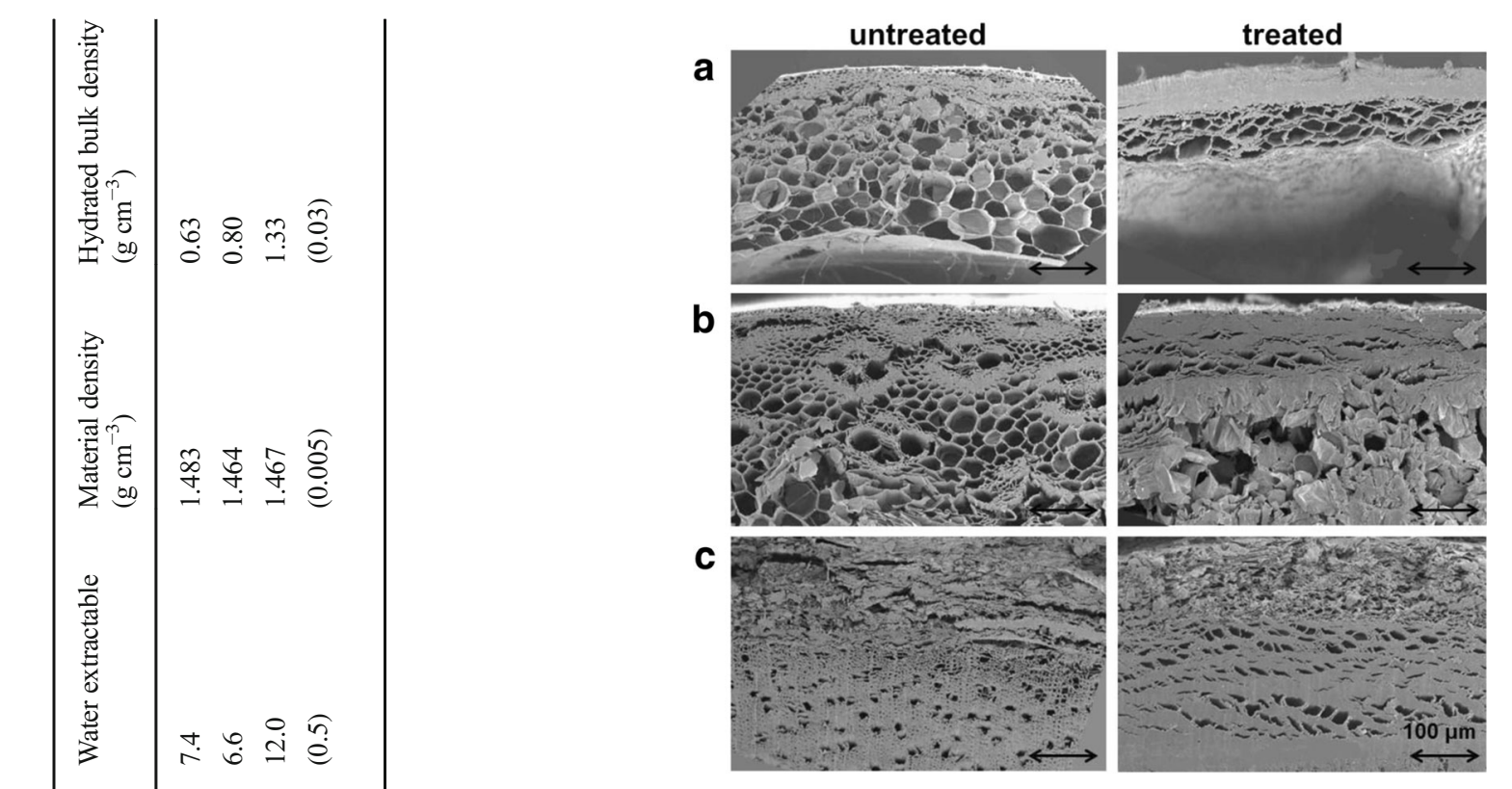

Fig. 2 Scanning electron micrographs of untreated and treated stem cross sections of biomass materials: a wheat straw, $\mathbf{b}$ Miscanthus and $\mathbf{c}$ willow

at the longest treatment time was slightly higher than that of the two grassy species. This is reflected in the fitted parameters in Table 2, which indicate that apparently a smaller fraction of the willow material experiences effective physicochemical deconstruction during hydrothermal treatment ( $p=0.35$ ) compared to both straw and Miscanthus $(p=0.58$ and 0.55$)$. This is consistent with the presence of a more chemically resistant cell wall structure in willow, as evidenced from the solubilisation profiles in Fig. $1 \mathrm{~b}$.

The most surprising observation from the experimental data was that the critical milling times for untreated and intermediate pretreated willow were considerably lower than for the equivalent samples of straw or Miscanthus, where these grassy species might have been expected to show poorer mechanical properties than the woody willow material. This differentiation is reflected in the model parameters, where the

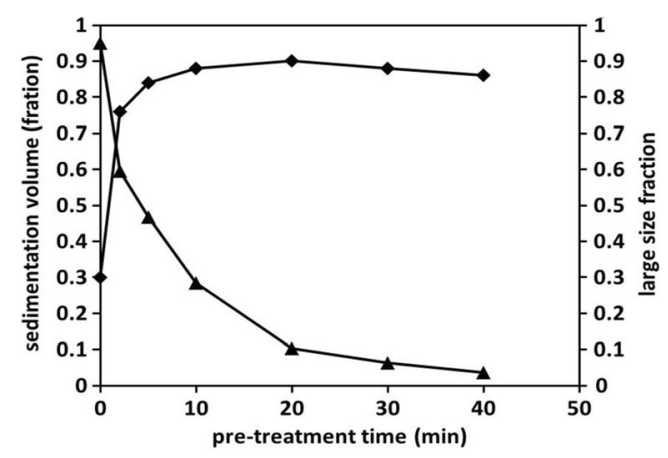

Fig. 3 Disintegration behaviour of willow biomass under a standardised wet-milling time of $10 \mathrm{~min}$, following different times of hydrothermal pretreatment: fractional sedimentation volume (filled diamond), proportion of large particle (>1 mm) fraction (filled triangle) 
material integrity parameter $(m)$ is higher for the two grasses than for willow. These counterintuitive findings may partly be a consequence of the differences in particle geometries between the species, as indicated from the micrographs of the dispersions of pretreated samples. The images in Fig. 5 show that after 10 min milling, the predominant vascular tissues of the grass species had broken up to create particles with low lateral dimensions, of around 10-20 $\mu \mathrm{m}$, but relatively high longitudinal dimensions, up to $1 \mathrm{~mm}$, and hence high aspect ratio. Such disintegration behaviour would mean that the tissue fragments generated could entangle in the dispersion and cause capillary blockage. The longitudinal strength of such vascular fragments would also mean that a longer critical milling time would be required to achieve sufficient breakup in this dimension for smooth flow. In comparison, from Fig. 5, the predominant willow xylem tissue had broken up to form lower aspect ratio cellular fragments, again around 10-20 $\mu \mathrm{m}$ in the lateral dimension and mostly less than $200 \mu \mathrm{m}$ in the longitudinal dimension, which therefore would entangle less severely and therefore achieve flow through the capillary at shorter critical milling times.
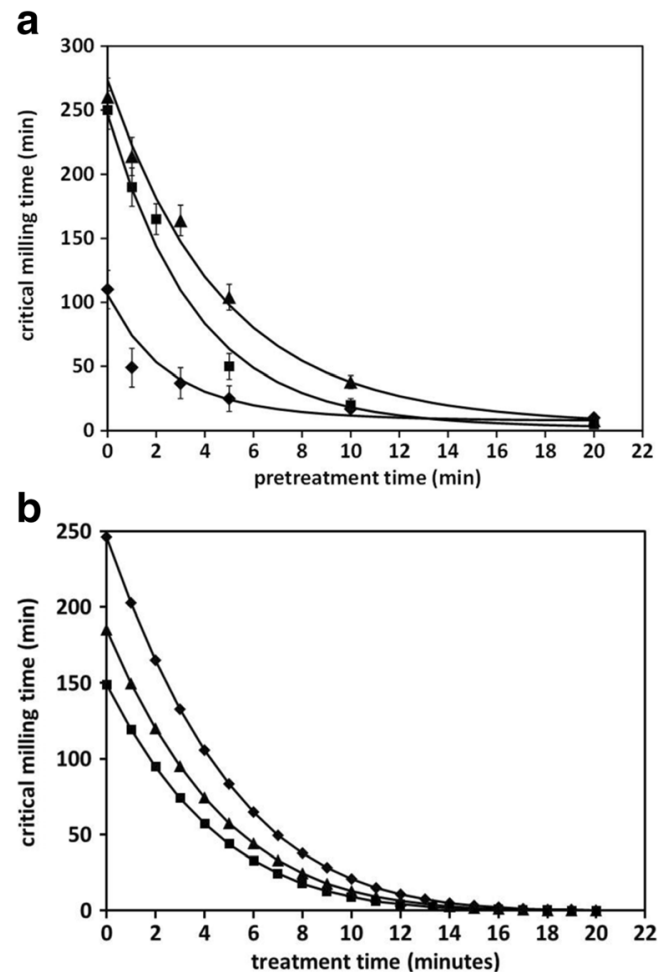

Fig. 4 a Experimental relationship between pretreatment time and wet ball-milling time required for reduction in particle size to achieve smooth capillary flow, for wheat straw (filled square), Miscanthus (filled triangle) and willow (filled diamond). Error bars are estimates of measurement error. Continuous lines are fits of data to the chemico-kinetic model. b Illustration of parameter sensitivity of the chemico-kinetic disintegration model, from Eqs. (2) and (3). Pretreatment rate constant $(k)=0.05 \mathrm{~min}^{-1}$, material integrity parameter $(m)=$ (filled square) 1.9 , (filled triangle) 2.0 , (filled diamond) $2.15 \mathrm{kmol} \mathrm{m}^{-3}$. Other parameters are fixed 


\subsection{Relationships between digestibility and material properties}

The experimental saccharification yields of the biomass dispersions at $24 \mathrm{~h}$ digestion time are shown graphically in Fig. 6 for different pretreatment times, with and without a 10-min milling time. These results clearly show the benefits gained from supplementary milling at all levels of pretreatment for all three biomass species. From this data, the intermediate 10-min pretreatment time was selected to provide the most representative comparisons between species, and the corresponding digestion kinetics were analysed in detail using the diffusion-limiting model, as described in Section 3. The fitted kinetic parameters are summarised in Table 3, where the yield at infinite time $\left(C_{\infty}\right)$ was set as the asymptotic experimental glucose yield found at $48 \mathrm{~h}$ digestion time for the 10 -min milled samples of each species. The diffusional parameter (n) was set at 0.3 (except 0.4 for unmilled willow), which indicated a high level of structural constraint for all tissue materials.

Comparative outputs from the kinetic analysis at $24 \mathrm{~h}$ digestion time showed that without milling, the pretreated willow gave the lowest glucose yield of $25 \%$, followed by unmilled Miscanthus at 38\%, with the highest yield of $51 \%$ for unmilled straw. However, this ordering changed dramatically following the progressive increase in milling time, from Table 3, where ultimately after 10 min milling, the pretreated willow achieved the greatest glucose yield of $77 \%$, therefore also showing the most impressive enhancement on milling. Conversely, the pretreated straw showed the least enhancement on milling, from 51 to $66 \%$ yield, despite the large reduction in critical milling time following pretreatment indicated from the disintegration model.

To understand these different extents of enhancement, it is helpful first to note that the trend in digestibilities of the three unmilled pretreated biomass materials appeared to follow the relative tissue density of the initial untreated materials, as visualised by electron microscopy, in Fig. 2. Here, intuitively, it would be expected that particles with a greater structural compactness would have lower enzyme accessibility, according to visual interpretation, in the order willow < Miscanthus < straw. This interpretation is supported more quantitatively from the measurement of the hydrated bulk densities of the untreated materials, which provides an indication of the amount of closed voids in the tissue structure. Values of $1.33,0.80$ and $0.67 \mathrm{~g} \mathrm{~cm}^{-3}$ were determined for willow, Miscanthus and straw, respectively, in Table 1, which were all lower than the absolute material densities measured by pycnometry, which were very similar for all three species. Willow clearly had the lowest internal void volume, reflecting the dense, closely packed, organisation of the xylem, phloem and cambrian tissue layers. Conversely, straw had the greatest void volume reflecting the more delicate compartmented nature of the sclerenchyma tissue of the plant stem, bounded by the outer vascular bundles, with Miscanthus having intermediate character. Further support for these inferences could be found from the settling behaviour of the respective pretreated sample dispersions, according to the comparative graph in Fig. 7a, where the pretreated unmilled willow material had the lowest fractional settled volume, followed by Miscanthus, then straw. The settling properties of the dispersions will also be strongly dependent on the hydrated densities of the biomass particles, as this material property will influence their buoyancy and hence the tendency of the particles to pack closely within the surrounding liquor under conditions of gravitational settling.

From these basic interpretations, it is suggested that the digestibility of the initial unmilled pretreated biomass particles might be correlated most simplistically with differences in tissue density and structural compactness between the three species. Following this logic, all three pretreated biomass species exhibited gains in digestibility after wet-milling, due to the increase in accessibility of the disintegrated tissue structures through creation of new surfaces. However, the relative enhancements observed on milling may again depend on the differences in tissue textures between species, as manifested in the differing particle geometries, which may again be inferred by observation of particle settling behaviour. The settled volumes of the pretreated materials all increased as a result of milling, from Fig. 7a, as smaller particles would be suspended more effectively in the surrounding liquor. As anticipated, from Fig. 7b, an overall positive correlation was seen between the measured settled volume and the corresponding enzyme digestibility, as the surface area accessible for enzyme interaction would increase with reduction in particle size. However,

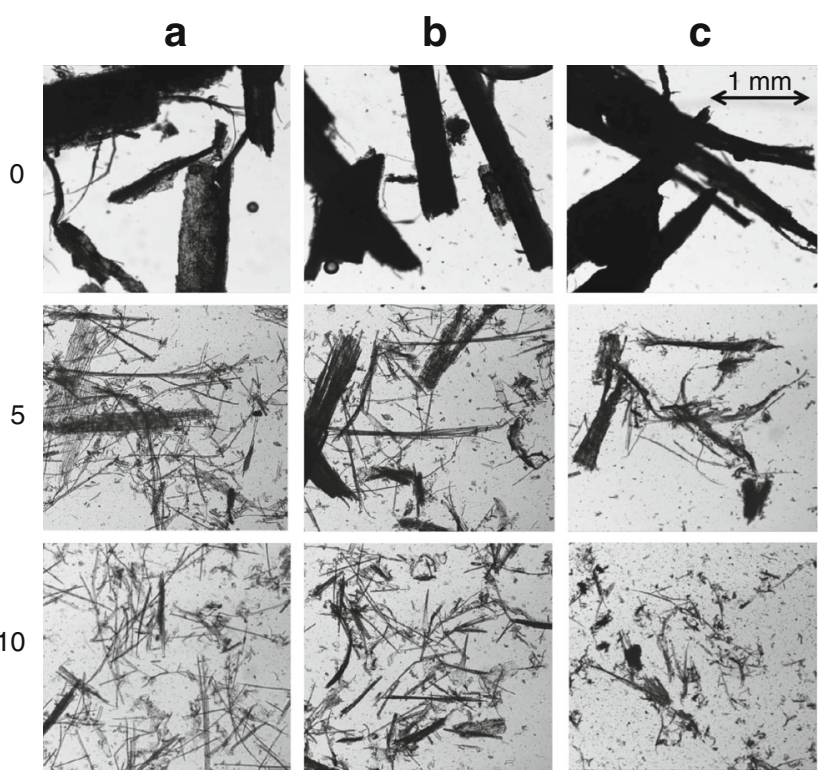

Fig. 5 Optical micrographs of biomass materials hydrothermally treated for 10 min duration, subjected to different times of supplementary wet ballmilling (0, 5 and $10 \mathrm{~min})$ : a wheat straw, b Miscanthus and $\mathbf{c}$ willow. Scale bar $=1 \mathrm{~mm}$ for all images 


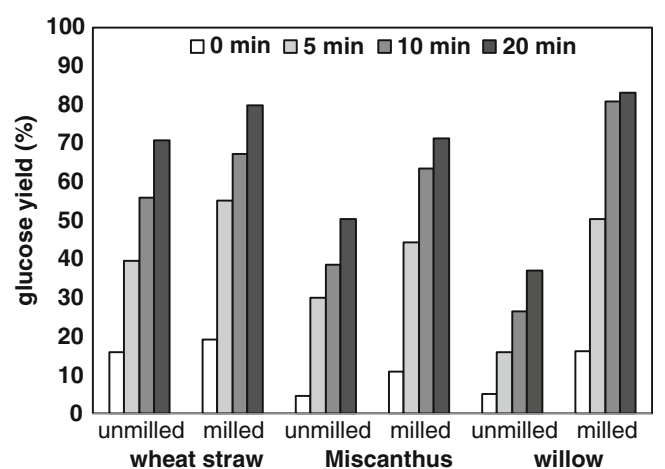

Fig. 6 Glucose yield from enzyme digestion of biomass materials subjected to different pretreatment times, with no supplementary milling or supplementary milling for $10 \mathrm{~min}$. Light to dark bars = pretreatment times of $0,5,10$ and $20 \mathrm{~min}$

although the curves for straw and Miscanthus followed approximately the same trajectory, this was offset significantly to lower gradient compared to the curve for willow. As a possible explanation of these differences, it is firstly considered that the relatively high hydrated density and lower aspect ratio characteristics of the willow fragments means that disintegration must be continued to a comparatively small particle size in order to achieve a fully suspended particle network and high settled volume. Such small particles clearly have high accessibility, resulting in a high overall gain in digestibility. In contrast, for both Miscanthus and straw, the disintegration of the stem tissue due to milling results in the development of fragments with relatively lower hydrated density and with higher aspect ratio. Such rod-like particles will form a more effective suspended network and will achieve a higher settled volume, reached at shorter milling times. The low lateral dimensions of such particles may also result in the more rapid achievement of a high accessible surface area. With continued milling, the longitudinal dimensions of the vascular fragments may be reduced, although this may possibly have a negative effect on the network suspension, reflected in the limited further gain in settled volume, as indicated in Fig. 7a. The rodlike nature of these structures may also mean that further reduction of longitudinal dimensions would achieve limited additional gain in accessible surface area and in digestion yield.

$\mathrm{X}$-ray diffraction measurements showed that some decrystallisation of the cellulose fibrils in all three biomass species was caused initially by hydrothermal treatment, from Table 4, which has been observed in earlier studies [37]. However, no further loss of crystallinity had occurred due to wet ball-milling, and in fact, there was a moderate increase in cellulose crystallinity, similar for all three biomass species, probably as a result of water-induced recrystallisation (hornification) due to the additional drying step required for XRD measurement [38]. The increase in digestibility brought about by wet-milling cannot therefore be ascribed to any additional mechanically induced decrystallisation of the cellulose fibrils and must therefore be a result of the largerscale structural and textural changes causing an increase in fibril accessibility.

\subsection{Disintegration model parameters and relation with digestibility}

As discussed, the apparently lower material integrity parameter $(m)$ of willow compared to straw and Miscanthus may partly be a consequence of differences in particle aspect ratios as manifested by the capillary flow measurements used to follow disintegration behaviour. However, the lower value of this parameter does reflect the ultimately high extent of disintegration of the willow biomass species that could be reached by milling following pretreatment, and the high digestibility that could therefore be attained. The higher material integrity parameters for straw and Miscanthus may also intuitively reflect the greater longitudinal strength of the separated vascular fragments of these materials, which limited the achievement of capillary flow of the respective milled dispersions.

From the model outputs in Table 2, as discussed earlier, apparently a lower proportion of the willow biomass undergoes effective chemical deconstruction $(p=0.35)$, compared to straw and Miscanthus tissue ( $p=0.58$ and 0.55). This may be interpreted as an indication of a higher residual recalcitrance of the willow cell wall structure following pretreatment, as suggested from the solubilisation profiles in Fig. 1b. Consequently, cellulose enzymatic hydrolysis may still be inhibited by interferences from other chemical functionalities, in addition to the low accessibility of the initial willow particles. However, from a mechanical perspective, the hydrothermal pretreatment of willow does result in sufficient loss of

Table 3 Digestion kinetics of $0.5 \%$ dispersions of pretreated biomass milled for different times

\begin{tabular}{lccccccccc}
\hline Material & \multicolumn{1}{c}{ Straw } & \multicolumn{4}{c}{ Miscanthus } \\
\hline Milling time (min) & 0 & 5 & 10 & 0 & 5 & 10 & 0 & 5 \\
Experimental yield at 24 h (\%) & 51 & 63 & 67 & 39 & 61 & 64 & 26 & 56 & 81 \\
Rate constant $(r)\left(\mathrm{h}^{-1}\right)$ & 0.015 & 0.056 & 0.075 & 0.007 & 0.057 & 0.077 & $0.002 *$ & 0.013 & 0.054 \\
$R^{2}$ of fit & 0.991 & 0.987 & 0.979 & 0.996 & 0.988 & 0.926 & 0.923 & 0.986 & 0.980 \\
Modelled yield at 24 h $(\%)$ & 51 & 64 & 66 & 38 & 60 & 62 & 25 & 57 \\
\hline
\end{tabular}

Kinetic fitting from Eq. (4). $n=0.3$ for all data, except $*$ where adequate fit required $n=0.4$ 
a

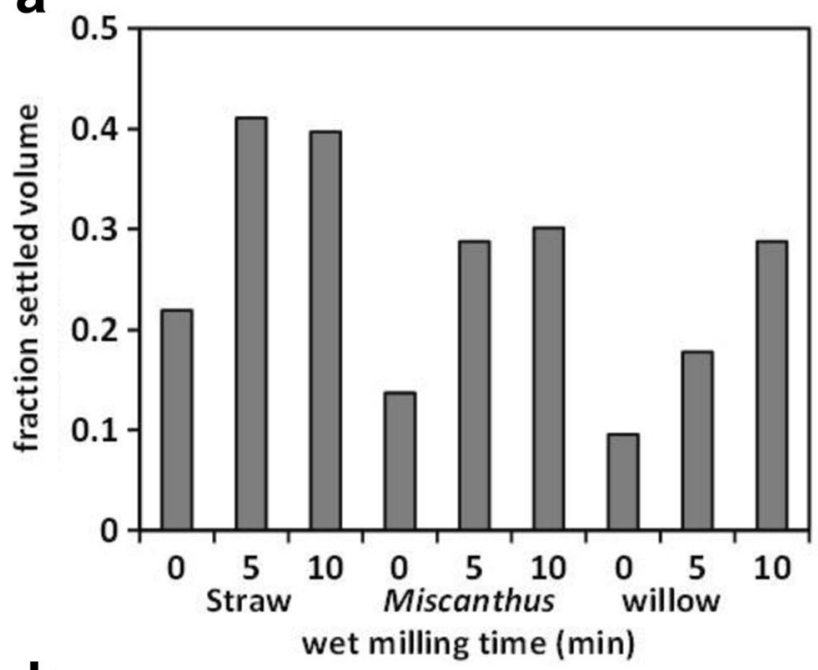

b

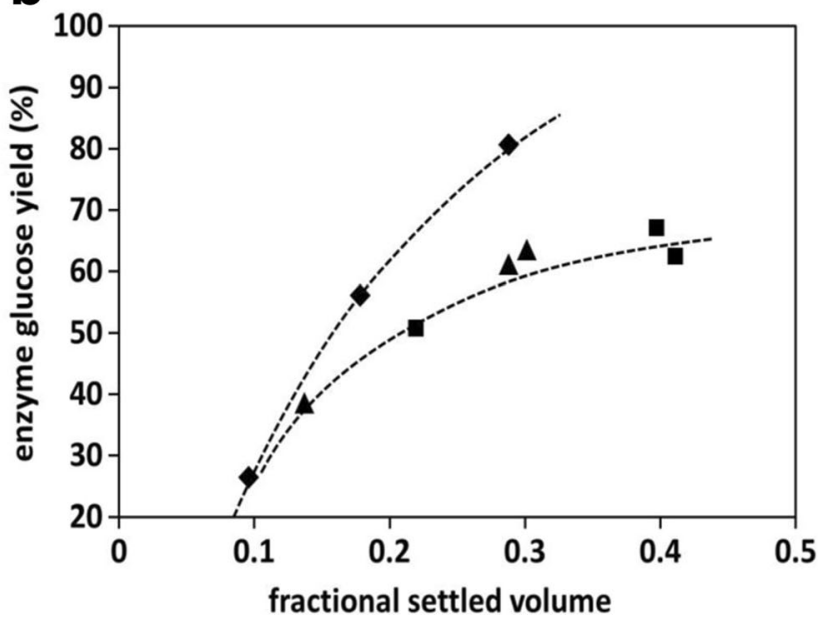

Fig. 7 a Settled volumes of straw, Miscanthus and willow biomass following pretreatment for $10 \mathrm{~min}$, then subjected to ball-milling for 0 , 5 and 10 min durations. Dispersions at $0.5 \%$ solids content. b Relationships between glucose enzymatic yield and fractional settled volume for combined hydrothermal treatment followed by wet ballmilling, according to conditions from a. Wheat straw (filled square), Miscanthus (filled triangle), willow (filled diamond)

structural integrity to allow effective particle breakup under milling conditions. Ultimately, this more than compensates for any residual recalcitrance of the tissue material, provided that milling is continued to reach sufficiently small particle size.

The value of the external stress parameter $(\sigma)$ used in the fitting of the disintegration model reflects the high mechanical energies delivered under the wet ball-milling regime. This contrasts with the regime experienced during the dilute enzyme digestion assay, where the dispersed biomass particles experience much lower mechanical energy due to shaking in the laboratory incubator. Although particle breakup might occur simply as a result of this mild physical agitation, this would be a much slower process, as illustrated from the model, where a reduction of the stress parameter by /100 would increase critical milling time for fully pretreated willow from $10 \mathrm{~min}$ to an excessive time of $174 \mathrm{~h}$, compared to wet ballmilling. Therefore, in principle, even with pretreatment, the tissue particles of all three biomass species should be resistant to further disintegration under the timescales and conditions of the enzyme assay. However, it is clear that during digestion, there will be an increasing loss of material continuity due to the hydrolytic depolymerisation reactions taking place within the cell wall structure. This ongoing biochemical deconstruction would reduce the integrity parameter $(m)$ of the disintegration model, predicting a shortening of this exceptionally long timescale. In the future, elements of the Zhukov theory could be included in a more complete model for biomass digestion, accounting for these synergistic biochemical-mechanical interactions and their combined effect on enzyme kinetics.

\subsection{Implications for process optimisation}

In energy terms, a reduction in milling time required to achieve a target biomass surface area will translate directly as a saving in energy consumed, which can be predicted from the model. The laboratory ball-mill is ideal for control of experimental variables but is inefficient in process terms. However, other workers have cited energy consumptions using commercial wet disk-milling methods of around $2.1 \mathrm{MJ} / \mathrm{kg}$ in order to achieve surface areas of the order of $0.2 \mathrm{~m}^{2} / \mathrm{g}$ for untreated woody species, which could possibly be reduced only $0.07 \mathrm{MJ} / \mathrm{kg}$ by physico-chemical pretreatment, which is a $30 \times$ saving $[23,25]$. This compares with the modelling outputs from the current study, which from Fig. 4a indicate that with a 20 -min hydrothermal pretreatment the potential time/energy saving will be around $42 \times$ for straw, $34 \times$ for Miscanthus and $11 \times$ for willow. For an intermediate 10-min pretreatment, the savings are $14 \times, 8 \times$ and $7 \times$ for straw, Miscanthus and willow, respectively.
Table 4 Cellulose crystallinities in untreated and hydrothermally treated biomass materials

\begin{tabular}{llll}
\hline & Untreated (\%) & $\begin{array}{l}10 \text { min hydrothermally treated } \\
\text { only (dried once) }(\%)\end{array}$ & $\begin{array}{l}\text { Hydrothermally treated then } \\
\text { wet-milled (dried twice) (\%) }\end{array}$ \\
\hline Straw & 67 & 50 & 58 \\
Miscanthus & 66 & 49 & 55 \\
Willow & 69 & 54 & 61 \\
Est. error (+/-) & $(2)$ & $(2)$ & $(2)$ \\
\hline
\end{tabular}


Supplementary milling following pretreatment represents an additional process cost, so its value in terms of an overall process must be considered. This work shows that the gains in digestibility are greater for the woody willow biomass than for the grassy straw, which performs adequately without this supplementary step, with Miscanthus having intermediate behaviour. The data in Fig. 6 also shows that even with an extension of hydrothermal treatment time, it is not possible to achieve the highest saccharification yield for willow without supplementary milling. Fermentation inhibitor compounds also build up at longer hydrothermal treatment times, as well as an increase in glucose degradation, so a continued extension of this process step is not viable [26]. Therefore, in an industrial operation, supplementary wet-milling might be most usefully applied to woody feedstocks, where from Fig. 6 of this study, a realistic $20 \mathrm{~min}$ pretreatment, followed by milling, followed by $24 \mathrm{~h}$ digestion, could lead to an increase of $184 \mathrm{~g} / \mathrm{kg}$-biomass of glucose yield for willow (on initial biomass), with corresponding gains in ethanol yield. This compares with a $91 \mathrm{~g} / \mathrm{kg}$-biomass gain for Miscanthus and $35 \mathrm{~g} / \mathrm{kg}$-biomass gain for straw. From a calorific perspective, this represents an enhancement in energy yield of around $2.8 \mathrm{MJ} / \mathrm{kg}$-biomass for willow, compared to the use of $\sim 0.1 \mathrm{MJ} / \mathrm{kg}$-biomass for the supplementary milling step and compared to an energy of $1.8 \mathrm{MJ} / \mathrm{kg}$-biomass for hydrothermal treatment [39]. Equivalent gains of 1.4 and $0.54 \mathrm{MJ} / \mathrm{kg}$-biomass would be realised for Miscanthus and wheat straw, respectively.

This work has explored the use of specific material assays, such as the hydrated bulk density and settling measurements, and the direct measurement of disintegration characteristics through controlled milling. Such assays may sensibly reflect the characteristics of biomass of importance for saccharification efficiency, for guidance of feedstock selection and breeding programmes. The current results suggest that resistance to wet-state mechanical fatigue type processes is a key indicator of biomass performance, which can be modelled successfully by the current approach. The loss of mechanical durability through physico-chemical pretreatment is a complex product of both molecular and microscale biomass structure, dependent on both the chemical lability of cell wall functionalities and the overall architecture of biomass tissue residues.

\section{Conclusions}

A chemico-kinetic disintegration model has been used to describe the relationship between the extent of hydrothermal pretreatment and the critical milling time required for a reduction of biomass material to a specific particle size. The model confirms that significant energy savings in milling can be achieved by prior physico-chemical treatment and that the effective size reduction of biomass can lead to significant gains in digestibility. Differences in behaviour between biomass species are strongly dependent on tissue structure and organisation, which is reflected in model parameters. The work has shown that a woody biomass can be processed efficiently by these cooperative techniques to achieve high digestion yields, equivalent if not superior to yields from grass species.

The work has indicated that the vascular fragments of pretreated grassy species are resistant to longitudinal breakup, which gives rise to a high material integrity parameter in the model, in contrast with the predominant secondary xylem fragments of woody species. Therefore, model behaviour does not fully correlate with observed trends in digestibility, as such grassy vascular fragments have high surface area and high accessibility. The model outputs indicate that a lower proportion of the woody willow biomass undergoes effective deconstruction under physico-chemical pretreatment conditions compared to the grassy species, which is consistent with its greater observed chemical resistance. The model also sensibly predicts that biomass disintegration will be slow under a low energy mixing or shaking regime, although this does not currently account for the ongoing loss of material integrity resulting from enzymatic hydrolysis. These findings suggest further opportunities for refinement of the model in the future.

Acknowledgements The authors would like to thank Dr. Sarah Purdy at IBERS, Aberystwyth, UK, and Jennifer Cunniff at Rothamsted Research Station, UK, for the supply of biomass feedstocks.

Funding The research was supported by the Biotechnology and Biological Sciences Research Council Sustainable Bioenergy Centre (BSBEC), under the programme for 'Lignocellulosic Conversion To Ethanol (LACE)' [Grant Ref: BB/G01616X/1].

Open Access This article is distributed under the terms of the Creative Commons Attribution 4.0 International License (http:// creativecommons.org/licenses/by/4.0/), which permits unrestricted use, distribution, and reproduction in any medium, provided you give appropriate credit to the original author(s) and the source, provide a link to the Creative Commons license, and indicate if changes were made.

\section{References}

1. Schädel C, Blöchl A, Richter A, Hoch G (2010) Quantification and monosaccharide composition of hemicelluloses from different plant functional types. Plant Physiol Biochem 48:1-8

2. Lewis NG, Yamamoto E (1990) Lignin: occurrence, biogenesis and biodegradation. Annu Rev Plant Physiol Plant Mol Biol 41:455-496

3. Mosier N, Wyman C, Dale B, Elander R, Lee Y-Y, Ml H, Ladisch $M$ (2005) Features of promising technologies for pretreatment of lignocellulosic biomass. Bioresour Technol 96:673-686

4. Ingram T, Wörmeyer K, Lima JCI, Bockemühl V, Antranikian G, Brunner G, Smirnova I (2011) Comparison of different pretreatment methods for lignocellulosic materials. Part I: conversion of rye straw to valuable products. Bioresour Technol 102:5221-5228

5. Zhang Y-HP, Lynd LR (2004) Toward an aggregated understanding of enzymatic hydrolysis of cellulose: noncomplexed cellulase systems. Biotechnol Bioeng 88(7):797-782

6. Hong J, Ye X, Zhang YHP (2007) Quantitative determination of cellulose accessibility to cellulase based on adsorption of a 
nonhydrolytic fusion protein containing CBM and GFP with its applications. Langmuir 23:12535-12540

7. Zhu L, O'Dwyer JP, Chang VS, Granda CB, Holtzapple MT (2008) Structural features affecting biomass enzymatic digestibility. Bioresour Technol 99:3817-3828

8. Grethlein HE, Converse AO (1991) Common aspects of acid prehydrolysis and steam explosion for pretreating wood. Bioresour Technol 36:77-82

9. Chundawat SPS, Donohoe BS, Sousa L, Elder T, Agarwal UP, Lu F, Ralph J, Himmel ME, Balana V, Dale BE (2011) Multi-scale visualization and characterization of lignocellulosic plant cell wall deconstruction during thermochemical pretreatment. Energy Environ Sci 4:973-984

10. Esteghlalina A, Hashimoto AG, Fenske JJ, Penner MH (1997) Modelling and optimisation of the dilute sulphuric-acid pretreatment of corn stover, poplar and switchgrass. Bioresour Technol 59:129-136

11. Ray MJ, Brereton MJB, Shield I, Karp A, Murphy RJ (2012) Variation in cell wall composition and accessibility in relation to biofuel potential of short rotation coppice willows. Bioenergy Res 5(3):685-698

12. Abramson M, Shoseyov O, Shani Z (2010) Plant cell wall reconstruction toward improved lignocellulosic production and processability. Plant Sci 178:61-72

13. Dien BS, Jung H-JG, Vogel KP, Casler MD, Lamb JFS, Iten L, Mitchell RB, Sarath G (2006) Chemical composition and response to dilute-acid pretreatment and enzymatic saccharification of alfalfa, reed canary grass, and switchgrass. Biomass Bioenergy 30:880-891

14. Pedersen M, Meyer AS (2009) Influence of substrate particle size and wet oxidation on physical surface structures and enzymatic hydrolysis of wheat straw. Biotechnol Prog 25(2):399-408

15. Shuhaida H, Balan V, Takriff MS, Hassan O, Jahim J, Dale BE (2013) Performance of AFEX pretreated rice straw as source of fermentable sugars: the influence of particle size. Biotechnol Biofuels 6:40-56

16. Fan LT, Lee Y-H, Beardmore DH (1980) Mechanism of the enzymatic hydrolysis of cellulose: effects of major structural features of cellulose on enzymatic hydrolysis. Biotechnol Bioeng 22:177-I99

17. Lin Z, Huang H, Zhang H, Shzng L, Yan L, Chen J (2010) Ballmilling pretreatment of corn stover for enhancing the efficiency of enzyme hydrolysis. Appl Biochem Biotechnol 162:1872-1880

18. Hoeger IC, Nair SS, Ragauskas AJ, Deng Y, Rojas OJ, Zhu JY (2013) Mechanical deconstruction of lignocellulosic cell walls and their enzymatic saccharifcation. Cellulose 20:807-818

19. Lee S-H, Teramoto Y, Endo T (2009) Enzymatic saccharification of woody biomass micro/nanofibrillated by continuous extrusion process: 1 - effect of additives with cellulose affinity. Bioresour Technol 100:275-279

20. Kelsey RG, Shafizadeh F (1980) Enhancement of cellulose accessibility and enzymatic hydrolysis by simultaneous wet milling. Biotechnol Bioeng 12:1205-1036

21. Mais U, Esteghlalian AR, Saddler JN, Mansfield SD (2002) Enhancing the enzymatic hydrolysis of cellulosic materials using simultaneous ball milling. Appl Biochem Biotechnol 1(9):815-832

22. Gonzalez R, Jameel H, Chang H, Treasure T, Pirraglia A, Saloni D (2011) Thermo-mechanical pulping as a pretreatment for agricultural biomass for biochemical conversion. Bioresources 6(2):1599-1614

23. Zhu W, Zhu JY, Gleisner R, Pan XJ (2010) On energy consumption for size-reduction and yields from subsequent enzymatic saccharification of pretreated lodgepole pine. Bioresour Technol 101(8): 2782-2792

24. Hosseini SA, Shah N (2009) Multiscale modelling of hydrothermal biomass pretreatment for chipsize optimization. Bioresour Technol 100:2621-2628

25. Zhu JY, Wang GS, Pan XJ, Gleisner R (2009) Specific surface to evaluate the efficiencies of milling and pretreatment of wood for enzymatic saccharification. Chem Eng Sci 64:474-485

26. Rasmussen H, Sørensen HR, Meyer AS (2014) Formation of degradation compounds from lignocellulosic biomass in the biorefinery: sugar reaction mechanisms. Carbohydr Res 385:45-57

27. Zhukov SN (1965) Kinetic concept of the strength of solids. Int J Fract Mech 1:311-323

28. Regel VR, Leksovsky AM (1967) A study of fatigue within the framework of the kinetic concept of fracture. Int J Fract Mech 3(2):99-109

29. Ibbett R, Su Y, Renfew H, Phillips D, Taylor J (2009) Evaluation of the mechanical properties of lyocell textile materials crosslinked with 2,4-diacrylamidobenzenesulfonic acid under swollen and non-swollen conditions. J Appl Polym Sci 114:2116-2127

30. Ibbett R, Gaddipati S, Greetham D, Hill S, Tucker G (2014) The kinetics of inhibitor production resulting from hydrothermal deconstruction of wheat straw studied using a pressurised microwave reactor. Biotechnol Biofuels 7:45-58

31. Overend RP, Chornet E (1987) Fractionation of lignocellulosics by steam-aqueous pretreatments. Philos Trans R Soc Lond 321:523-536

32. Sluiter A, Hames B, Ruiz R, Scarlata C, Sluiter J, Templeton D, Crocker D (2011) Determination of structural carbohydrates and lignin in biomass. NREL Technical Report/TP-510-42618

33. Hatfield RD, Grabber J, Ralph J, Brei K (1999) Using the acetyl bromide assay to determine lignin concentrations in herbaceous plants: some cautionary notes. J Agric Food Chem 47:628-632

34. Ibbett R, Gaddipati S, Hill S, Tucker G (2013) Structural reorganisation of cellulose fibrils in hydrothermally deconstructed lignocellulosic biomass and relationships with enzyme digestibility. Biotechnol Biofuels 6:33-48

35. Carrillo F, Lis MJ, Colom X, Lopez-Mesas M, Valldeperas J (2005) Effect of alkali pretreatment on cellulase hydrolysis of wheat straw: kinetic study. Process Biochem 40:3360-3364

36. Brunow G, Lundquist K (2010) Functional groups and bonding patterns in lignins (including lignin-carbohydrate complexes) chapter 7. In: Heitner C, Dimmel RD, Schmidt JA (eds) Lignins and lignans. CRC, Boca Raton

37. Driemeier C, Pimenta MTB, Rocha GJM, Oliveira MM, Mello DB, Maziero P, Goncalves AR (2011) Evolution of cellulose crystals during prehydrolysis and soda delignification of sugarcane lignocellulose. Cellulose 18:1509-1519

38. Chen Y, Wang Y, Wan J, Ma Y (2010) Crystal and pore structure of wheat straw cellulose fiber during recycling. Cellulose 17:329-338

39. Zhu JY, Pan XL (2010) Woody biomass pretreatment for cellulosic ethanol production: technology and energy consumption evaluation. Bioresour Technol 101:4992-5002 